\title{
Velocity Control of Autonomous Powered Wheelchair Based on Interaction Prediction of Surrounding Pedestrian*
}

\author{
Takuma ITO** and Minoru KAMATA** \\ **The University of Tokyo, \\ 7-3-1, Hongo, Bunkyo-ku, Tokyo 113-8656, Japan \\ E-mail: ito@sl.t.u-tokyo.ac.jp
}

\begin{abstract}
Although electric-powered mobility devices with autonomous locomotion functions are considered helpful in assisting the elderly with decreased abilities to go on outings, technologies for safe locomotion in an outdoor environment with pedestrians have not yet been researched sufficiently. This research focuses on the autonomous locomotion on sidewalks where pedestrians coexist. An interactive locomotion strategy involving velocity control is also discussed. Taking into consideration the characteristics of sidewalks, a velocity control method that considers the interaction prediction of surrounding pedestrians was proposed and implemented into our prototype intelligent powered wheelchair. Through an experiment on a sidewalk test course, the operations of the proposed system were verified.
\end{abstract}

Key words: Intelligent Powered Wheelchair, Autonomous Locomotion, Interaction Prediction, Collision Avoidance

\section{Introduction}

As Japan has become a Super Aged Society, enabling the elderly's continued mobility to allow for outings is a major issue that is closely related to the maintenance of health. Electric powered mobility devices can help the elderly who have difficulty walking and participating in social activities ${ }^{(1)}$. For the elderly with the decreased mobility abilities, various types of mobility scooters have been developed. However, decreased cognitive abilities and judgment as a result of aging sometimes make it impossible for users of mobility aid to drive, even if they could drive previously. For aged users with decreased cognitive abilities, intelligent low-speed vehicles that have autonomous locomotion functions can be helpful.

Thus far, researchers have developed several kinds of intelligent low-speed vehicles that have autonomous support functions. Some researches have smartened commercial powered wheelchairs with sensors and computers such as ICW ${ }^{(2)}$ developed by Kayama et al. and TAO Aicle ${ }^{(3)}$ developed by Matsumoto et al. Other researchers have developed fundamentally novel intelligent low-speed vehicles called "Personal Mobility" to enhance the mobility of the elderly. MOBIRO ${ }^{(4)}$ is one such new low-speed vehicle, and it offers autonomous locomotion ${ }^{(5)}$. Previous studies developed the technologies necessary for autonomous locomotion, such as localization and object detection, and achieved safe autonomous locomotion on a certain level. As the environments envisioned for the usage of intelligent low-speed vehicles for the elderly vary widely, development of further technologies that are adapted to various situations is essential in order to put these kinds of 
vehicles into practical use. In this respect, the smooth autonomous locomotion of intelligent low-speed vehicles in public areas where pedestrians coexist, has not yet been examined well. Although technologies related to autonomous locomotion in a dynamic situation crowded with pedestrians have been developed in the research field of service robots and the "Real World Robot Challenge" such as the "Tsukuba Challenge" (6), these technologies assumed the use of unmanned robots whose locomotion priorities are lower than those of the surrounding pedestrians. As a result, existing unmanned robots mainly focus on uniform locomotion strategies, such as avoiding all traffic participants or always stopping for them. The most significant difference between intelligent mobility aids and unmanned service robots is the existence of its user; therefore, the locomotion priority of intelligent mobility aids needs to be equivalent to that of pedestrians. In order to realize autonomous locomotion with equivalent locomotion priority, intelligent mobility aids must be able to sense the surroundings, predict the situation, and behave adequately as well as pedestrians. In these respects, interactive locomotion strategy based on predictions of situations has not been adequately discussed. Therefore, this study focused on smooth autonomous locomotion on a sidewalk. This study also examines the interactive locomotion strategies based on the interaction prediction of surrounding pedestrians.

\section{Interaction Prediction of Surrounding Pedestrian}

\subsection{Assumed driving environment}

Figure 1 shows the appearance of a typical sidewalk, which is our target locomotion environment. Pedestrians, wheelchair users, mobility scooter users, and bicycle riders travel on common sidewalks, although bicycle riders have limited permission for most areas. Because their locomotion velocities vary widely as shown in Fig. 2, interactions such as overtaking by changing lanes, making a sufficient margin for safely overtaken, and avoiding oncoming traffic participants, happen very often. In addition, there are no defined rules for such interactions in this environment, although there is unspoken etiquette for vulnerable road users. In this situation, traffic participants usually control their self-locomotion and determine the timing of the execution of some kinds of interactions by paying attention to their surroundings continuously. Taking into consideration these actual situations, intelligent low-speed vehicles must act appropriately based on the predictions related to interactions between surroundings.

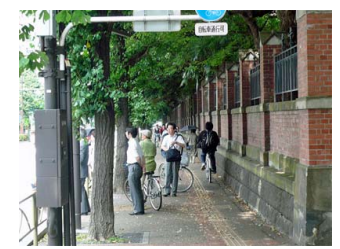

Fig. 1 Appearance of sidewalk

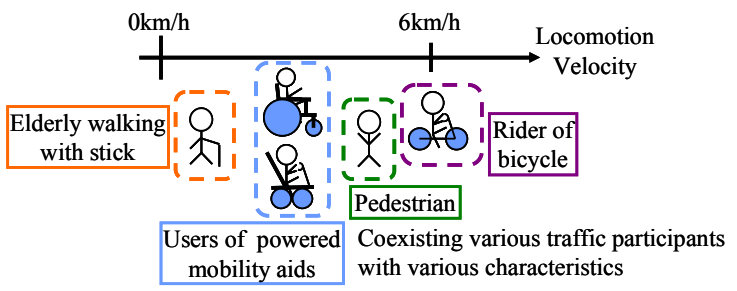

Fig. 2 Coexisting various traffic participants

\subsection{Existing research on prediction}

As for the locomotion strategy based on predictions, Hosoda et al. developed a novel collision avoidance algorithm for a human-symbiotic robot ${ }^{(7)}$. Their algorithm used the positions, volumes, and velocity vectors of surrounding pedestrians, and predicts the locomotion of surrounding pedestrians. As a result of the motion prediction based on the observed velocity vectors, their method realized collision avoidance even in a dynamic situation crowded with pedestrians. However, this method targets the situation where the velocity vectors of robot and traffic participants apparently cross each other. For example, if the velocity vectors apparently cross in the situation shown in Fig. 3, their method can predict the locomotion of pedestrians and avoid collisions safely. On the other hand, in the situation shown in Fig. 4, the observed velocity vectors do not appear to cross, and the 
pedestrian in front would change his route after a while. If the robot kept on moving until the pedestrian in front changed lanes, the situation would become so dangerous that the robot might need to make an emergency stop. Emergency stops are a severe problem for mobility aids, though they are not so critical for unmanned robots. One of the solutions for preventing emergency stops is proximity adjustment through velocity control such as deceleration and acceleration in the early phase while predicting future possible interactions. In this way, taking into consideration not only the current observed situation but also predicting future possible interactions seems necessary for effective interactive locomotion strategy on sidewalks. This study focuses on changing lanes as an example of interactions on sidewalks. Further, an interactive locomotion strategy of velocity control through interaction prediction of changing lanes is proposed.
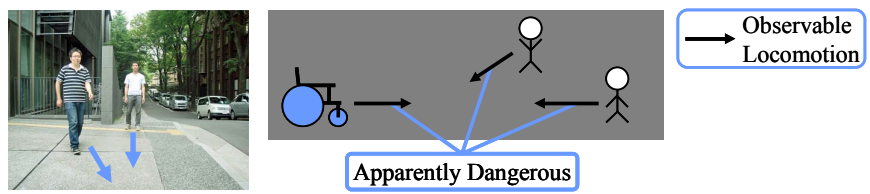

Fig. 3 Apparently dangerous situation
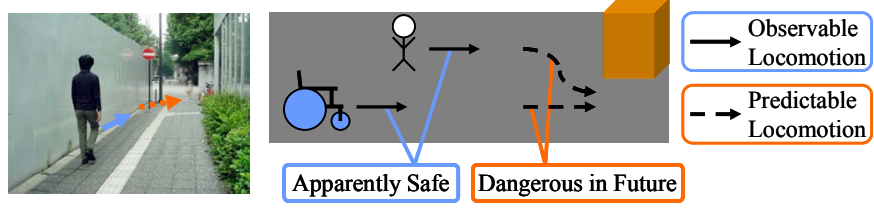

Fig. 4 Apparently safe but potentially dangerous situation

Some interaction predictions for pedestrians have been developed in some research projects. For example, Ziebart et al. ${ }^{(8)}$ researched the planning-based prediction method for pedestrian locomotion. Similarly, Chen et al. ${ }^{(9)}$ developed a method of pedestrian behavior prediction based on matching with classified motion patterns. The characteristics of these methods are dependencies on certain spaces and the stored data related to them that have attractive areas, such as lounges, kitchens, escalators, and so on. As for prediction methods that are independent of certain spaces, Ohki et al. ${ }^{(10)}$ developed a collision avoidance method for rescue robots. As their method used predictions of interactions between evacuees, our desired method was similar to theirs on some points. However, as their method assumed disastrous situations such as earthquakes, the surrounding pedestrians were generally in a panicked state. The above methods have good predictive accuracy because of the dependencies on a certain space or a certain pedestrian mental state. Because our assumed environments were simple sidewalks where various traffic participants with various conditions coexisted, such good accuracy could not be expected. Therefore, this study did not aspire to achieve precise interaction prediction with a small margin; rather, it strived to realize rough interaction prediction with a large margin. To be more precise, this study aimed at the rough prediction of interactions between pedestrians and objects by estimating the future arrival point on the basis of the current observed position and the velocity. Subsequently, the interactive locomotion strategy of velocity control to avoid potential future collision with rough interaction prediction, as shown in Fig. 5, was implemented in our prototype intelligent powered wheelchair for verification. Through the operation confirmation of the implemented system, the effectiveness of the proposed method would be verified. 

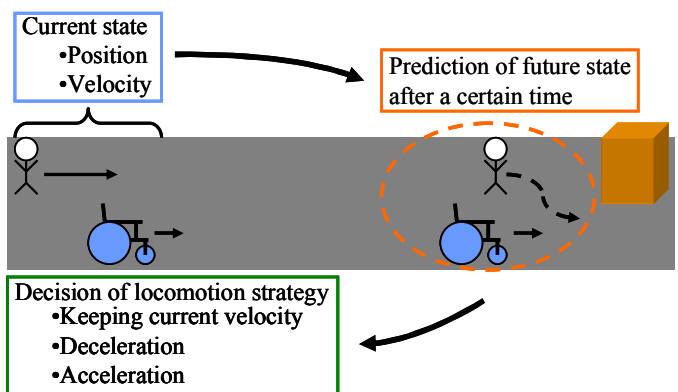

Fig. 5 Schematic of the interactive locomotion strategy of velocity control based on prediction

\section{Implementation}

\subsection{Prototype intelligent powered wheelchair}

Figure 6 shows the prototype intelligent powered wheelchair used in this study. This vehicle has two laser range sensors ("UTM-30LX," made by HOKUYO AUTOMATIC) and can detect static objects and dynamic traffic participants. In addition, based on the time-series measurement of the positions of traffic participants, the vehicle estimates their velocity vectors. As for the methods of recognizing surroundings and detecting traffic participants, various kinds of algorithms have been proposed up to now. In addition, some existing researches enhanced robustness of sensing methods based on the characteristics of the locomotion environments. The important point of this research is not detection method of surrounding traffic participants but locomotion strategy of the vehicle based on such information about surroundings. Thus, this research did not develop new algorithms but implemented simple algorithms of recognizing surroundings and detecting traffic participants. In addition, this research assumed the relatively simple sidewalk that had walls on one side, as shown in Fig. 1.

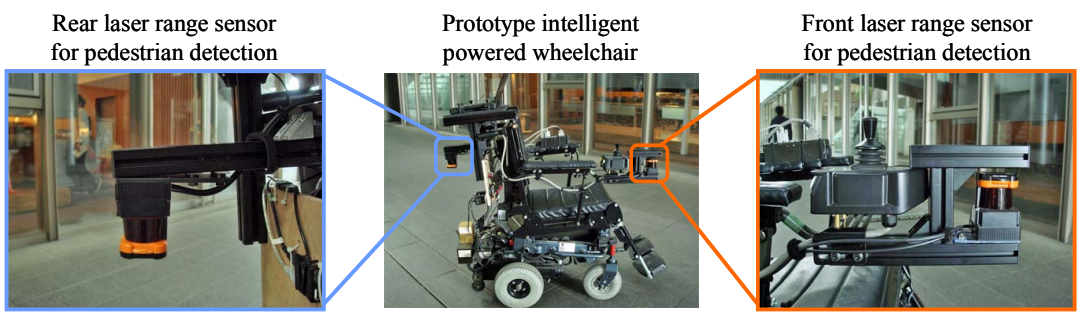

Fig. 6 Prototype intelligent powered wheelchair

First, for recognizing the surroundings, the vehicle detects the walls of the sidewalk by using the laser range sensors. Figure 7 shows the definitions of the variables used for wall detection. The wall detection component clusters the candidate points that satisfy the conditions expressed by following formulas:

$$
\begin{aligned}
& \left|\boldsymbol{v}_{i-1}\right|<\mathrm{D}_{\mathrm{wd}} \\
& \left|\boldsymbol{v}_{i}\right|<\mathrm{D}_{\mathrm{wd}} \\
& \frac{\boldsymbol{v}_{i-1} \cdot \boldsymbol{v}_{i}}{\left|\boldsymbol{v}_{i-1}\right|\left|\boldsymbol{v}_{i}\right|}>\cos \left(\theta_{\mathrm{wd}}\right)
\end{aligned}
$$

where $\boldsymbol{v}$ denotes the vector between the measured points, $\mathrm{D}_{\mathrm{wd}}$ denotes the threshold value of the distance between the measured points, and $\theta_{\mathrm{wd}}$ denotes the threshold value of the deviation angle between the vectors. 


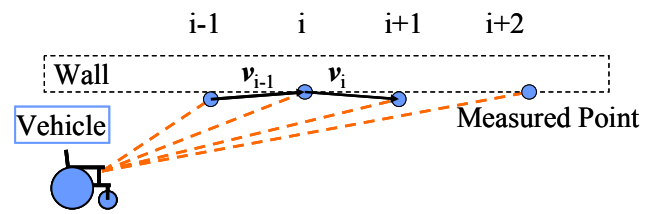

Fig. 7 Definition of the variables for wall detection

Then, the vehicle detects the surrounding objects by using the data of measured points that are not used for the wall detection. Figure 8 shows the definitions of the variables used for object detection. Using the remaining points not classified as part of the walls, this component gathers the candidate points that basically satisfy the following condition:

$$
d_{i}<\mathrm{D}_{\mathrm{cl}}
$$

where $d_{i}$ denotes the distance between the measured points, and $\mathrm{D}_{\mathrm{cl}}$ denotes the threshold value of the distance between the measured points. Although this simple detection method has disadvantages that it can not discriminate near objects and can not detect the object behind another object, these disadvantages are not so critical for the interaction prediction which uses information of distant objects. However, for practical use as a whole system, this detection method needs to be enhanced in the future. Existing method which detects the moving objects by time-series and probabilistic method seems effective for further robust object detection ${ }^{(11)}$.

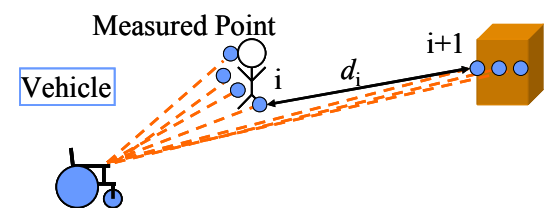

Fig. 8 Definition of the variables for object detection

Using the detected data, the vehicle roughly calculates the position and size of the objects. As the ratio of the shapes measured by the laser range sensor is less than half of all the shapes, the calculation formulations are simplified as follows:

$$
\begin{aligned}
& \boldsymbol{p}_{o}=\frac{\boldsymbol{p}_{\mathrm{s}}+\boldsymbol{p}_{e}}{2} \\
& S_{o}=\left|\frac{\boldsymbol{p}_{s}-\boldsymbol{p}_{e}}{2}\right|
\end{aligned}
$$

where $\boldsymbol{p}_{\boldsymbol{o}}$ denotes the position vector of the object, $\boldsymbol{p}_{\boldsymbol{s}}$ denotes the position vector of the start point of the object, $\boldsymbol{p}_{\boldsymbol{e}}$ denotes the position vector of the end point of the object, and $s_{o}$ denotes the size of the object.

In a direction perpendicular to the detected wall, the vehicle defines the lateral axis of the sidewalk, as shown in Fig. 9. Based on the lateral deviation between the static object and the surrounding pedestrian, the vehicle judges whether the static object is in front of the pedestrian or not.

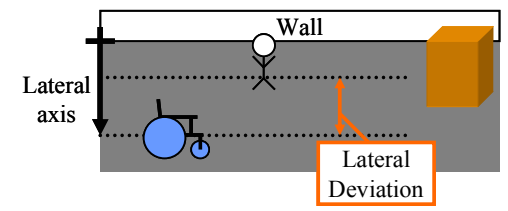

Fig. 9 Definition of the lateral axis of the sidewalk

\subsection{Interaction Prediction}

This study aimed at predicting pedestrian lane changing in the situations shown in Figs. 10 and 11 . These were the common situations in which pedestrians changed lanes to avoid the static obstacle in front of them, although the details regarding the position and the locomotion velocities differed. According to the Liu's research ${ }^{(12)}$, the average pedestrian 
margin distance to avoid static objects was approximately $2.9 \mathrm{~m}$. Thus, if the system can estimate the time required for a pedestrian to get $2.9 \mathrm{~m}$ in front of the objects, the system can roughly predict the future proximity between the vehicle and pedestrian when the pedestrian starts to change lanes. Based on the roughly predicted future proximity, the system decides whether the system keeps or changes the current velocity of the vehicle. As for the velocity control, the choice of either deceleration or acceleration requires consideration of the situation. For example, in situation 1 shown in Fig. 10, as the pedestrian in front may not grasp the situation, acceleration of the vehicle may cause a dangerous situation. In contrast, in situation 2 shown in Fig. 11, as both the pedestrian in rear and vehicle in front grasp the situation, the vehicle can avoid potential collision through either acceleration or deceleration. In this way, the vehicle needs to change the candidates of locomotion strategy to avoid potential dangerous situations by taking the situation into consideration.

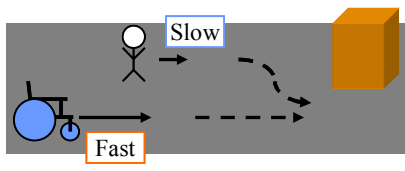

Fig. 10 Situation 1 where a slow pedestrian changes his course in the future

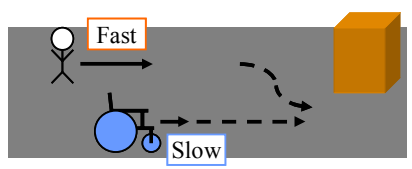

Fig. 11 Situation 2 where a fast pedestrian changes his course in the future

To numerically express the aforementioned idea, variables for the interaction prediction were defined, as shown in Fig. 12. $V_{P}$ denotes the current measured velocity of pedestrian, $V_{V}$ denotes the current measured velocity of vehicle, $D_{P}$ denotes the distance from pedestrian to static object, $D_{V}$ denotes the distance from vehicle to static object, $T_{r}$ denotes the time for the pedestrian to reach $3.0 \mathrm{~m}$ in front of the object, $P D_{P}$ denotes the predicted locomotion distance of the pedestrian for $T_{r}$, and $P D_{V}$ denotes the predicted locomotion distance of the vehicle for $T_{r}$. Although the average margin distance to avoid static objects was approximately $2.9 \mathrm{~m}$ according to Liu's research, this study assigned $3.0 \mathrm{~m}$ by rounding the value. The original point of the predicted locomotion distance for $T_{r}$ is the current position of the vehicle, whereas the original point of the distance to the object is the position of the static object. Relationships of these variables are expressed as follows:

$$
\begin{aligned}
& T_{r}=\frac{\left(D_{P}-3.0\right)}{V_{P}} \\
& P D_{P}=V_{P} \cdot T_{r}-\left(D_{P}-D_{V}\right) \\
& P D_{V}=V_{V} \cdot T_{r}
\end{aligned}
$$

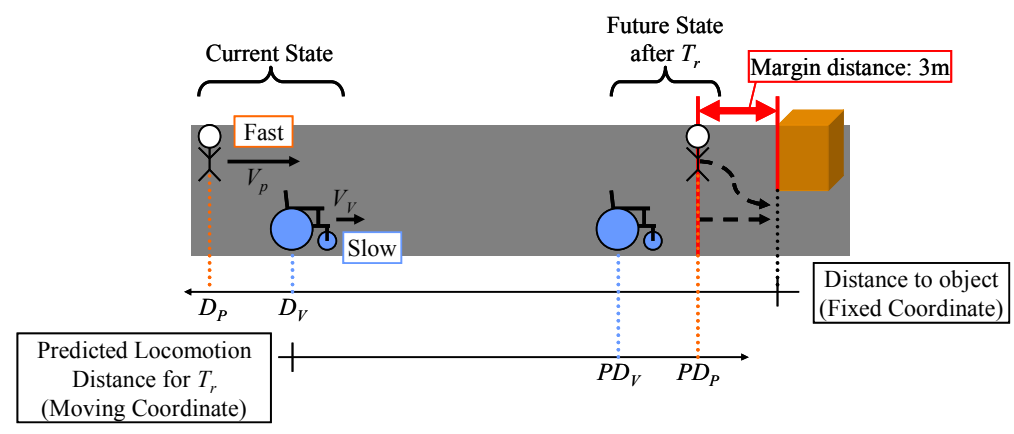

Fig. 12 Definitions of the variables for the interaction prediction

Using these variables, the system decides on the interactive strategy of velocity control: maintaining the current velocity, decelerating or accelerating. To make interactive strategy effective, good timing for strategy decision is necessary. Too early a decision to change strategy sometimes becomes useless, whereas too late a decision sometimes makes it impossible to avoid a potentially dangerous situation. Taking this issue into consideration, 
this research determined the conditions for changing the strategy of velocity control as follows:

$$
\begin{aligned}
& T_{r}<\mathrm{T}_{\text {limit }} \\
& \left|P D_{V}-P D_{P}\right|<\mathrm{D}_{\text {limit }}
\end{aligned}
$$

where $\mathrm{T}_{\text {limit }}$ denotes the limit time of $T_{r}$, and $\mathrm{D}_{\text {limit }}$ denotes the limit deviation between the distances of the vehicle and the pedestrian. If the situation satisfies these conditions, the system changes the velocity of the vehicle as follows:

$$
\mathrm{DV}_{\mathrm{V}}=\left\{\begin{array}{ccc}
V_{V}+0.28 & (\text { if } & \left.P D_{V}-P D_{P}>0\right) \\
V_{V}-0.28 & (\text { else })
\end{array}\right.
$$

where $\mathrm{DV}_{\mathrm{v}}$ denotes the desired velocity of the vehicle. In this research, adjustment velocity for acceleration and deceleration was assigned as $0.28 \mathrm{~m} / \mathrm{s}(1.0 \mathrm{~km} / \mathrm{h})$. $\mathrm{D}_{\text {limit }}$ was assigned as $1.2 \mathrm{~m}$, which is the longest longitudinal size of a powered wheelchair allowed in Japan. Because max acceleration of the vehicle used in this research is $0.42 \mathrm{~m} / \mathrm{s}^{2}(1.5 \mathrm{~km} / \mathrm{h} / \mathrm{s})$, it takes approximately $4.7 \mathrm{~s}$ to make margin distance of $1.2 \mathrm{~m}$ with acceleration of $0.28 \mathrm{~m} / \mathrm{s}$. Thus, $\mathrm{T}_{\text {limit }}$ was assigned as $5.0 \mathrm{~s}$ by rounding the value.

\section{Experiment for behavior confirmation of the system}

\subsection{Method}

The operation of the proposed system was verified by an experiment. Figure 13 shows the experimental course, which was a sidewalk mockup. The width was $2.0 \mathrm{~m}$, which is the narrowest width allowed for a sidewalk in Japan. In this course, the experimental collaborator behaved as a pedestrian. The velocity of the vehicle was $0.83 \mathrm{~m} / \mathrm{s}(3.0 \mathrm{~km} / \mathrm{h})$. Two kinds of situations were set up: situation 1 shown in Fig. 10 where a fast vehicle is overtaking a slow pedestrian, and situation 2 shown in Fig. 11 where a fast pedestrian is over taking a slow vehicle. The collaborator was told to set the locomotion velocity according to the situation.

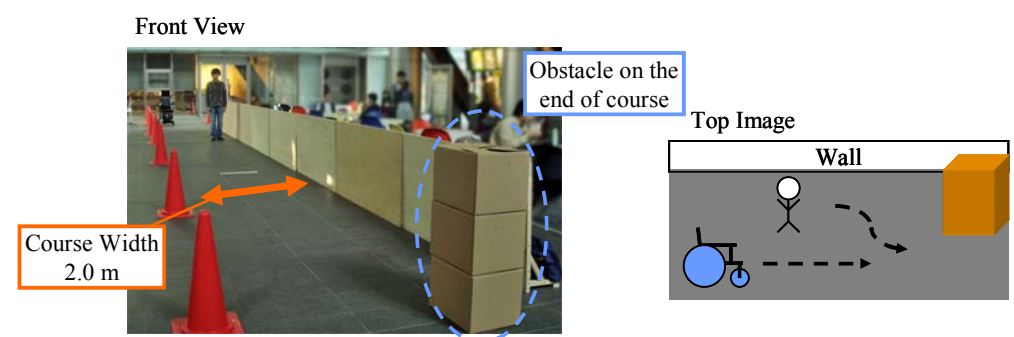

Fig. 13 Appearance and schematic of the experimental course

The experiment started with the vehicle proceeding autonomously by following the wall on the right side of the course, while the pedestrian walked on the left side of the course. Then, the rear traffic participant came up behind the front traffic participant. During this time, the vehicle sensed the position of the pedestrian and estimated $T_{r}$ and $P D \mathrm{~s}$ for the interaction prediction. When the situations satisfied the conditions expressed by Eqs. (10) and (11), the vehicle changed velocity depending on the situation.

\subsection{Results}

Figure 14 shows the time-series experimental results of $T_{r}$, predicted locomotion distances for $T_{r}$, distances to object and velocities for situation 1. Each line in red represents the results of the pedestrian, and each line in blue represents the results of the vehicle. Vertical orange lines represent the time when the situation satisfied the conditions expressed by Eqs. (10) and (11). At this point, the vehicle started to decelerate in this situation. As the distances to the objects show, the deviation of the distances to the objects decreases before 
deceleration. However, the deviation of the distances seems to stop decreasing after deceleration. To verify the effectiveness of the deceleration, the green circled part of the distances to the objects is picked up in Fig. 15. The dashed line in black represents the calculated distance from the vehicle to the objects if the vehicle maintained the velocity. As shown in the Fig. 15, the vehicle can make a sufficient margin to avoid the pedestrian changing lanes by deceleration. The final deviation between the measured distance of the actual vehicle and the calculated distance when the vehicle missed the pedestrian by the occlusion is approximately $2.0 \mathrm{~m}$, which is sufficiently larger than the allowed longitudinal size of the vehicle.

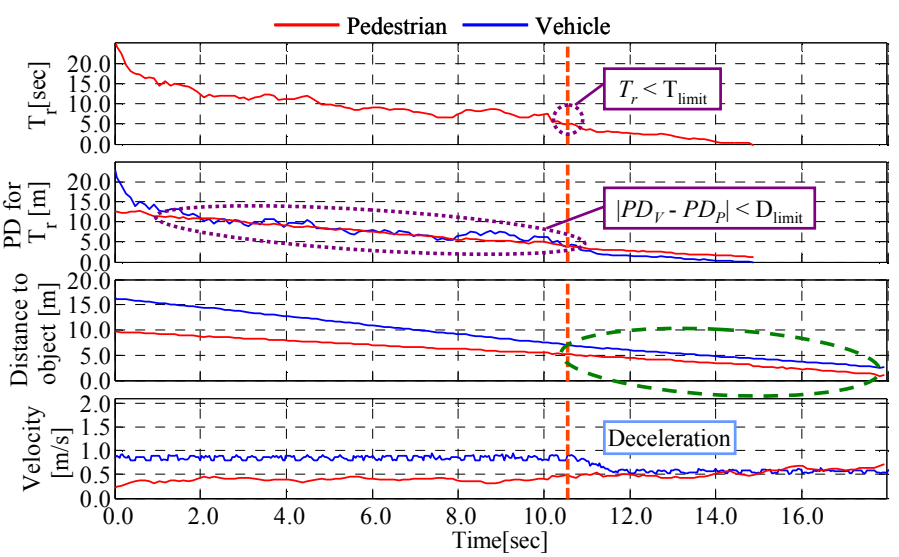

Fig. 14 Experimental results for situation 1

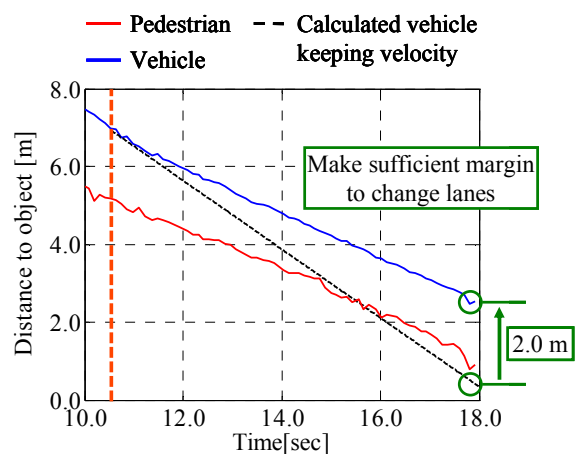

Fig. 15 Detailed transition of distance for situation 1

Similarly, Fig. 16 shows time-series experimental results for the acceleration case of situation 2. In this case, the predicted locomotion distance of the vehicle is larger than that of the pedestrian when the situation satisfied the conditions. As a result of this condition of predicted locomotion distance, the vehicle can create a sufficient margin distance by acceleration rather than deceleration. As in Fig. 15, Fig. 17 picks up the green circled part of the distance to the object shown in Fig. 16. Similar to the previous case, the vehicle can make a sufficient margin to avoid the pedestrian changing lanes by acceleration. The final deviation when the vehicle missed the pedestrian by the occlusion is approximately $1.2 \mathrm{~m}$. Because the passing time was shortened by the acceleration, the obtained margin distance is smaller than that by deceleration although this value also satisfies the required margin. 


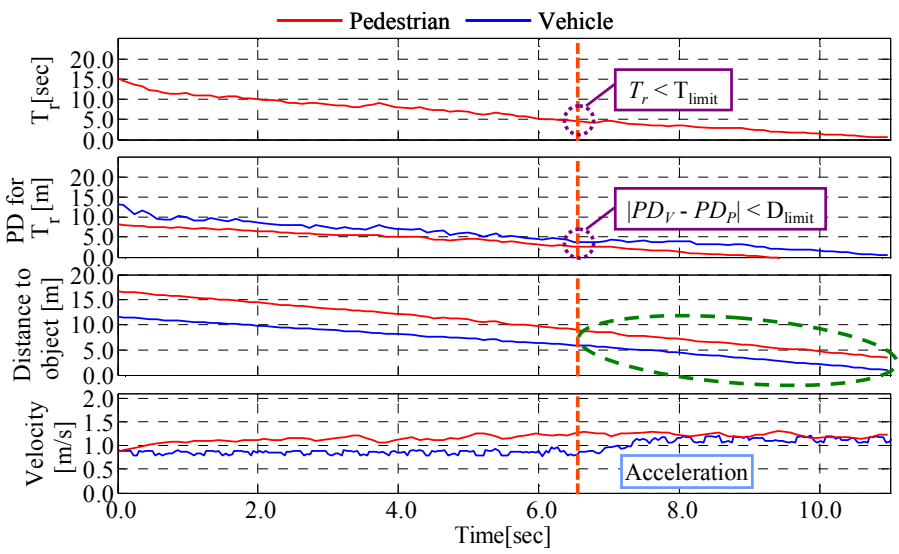

Fig. 16 Experimental results for the acceleration case of situation 2

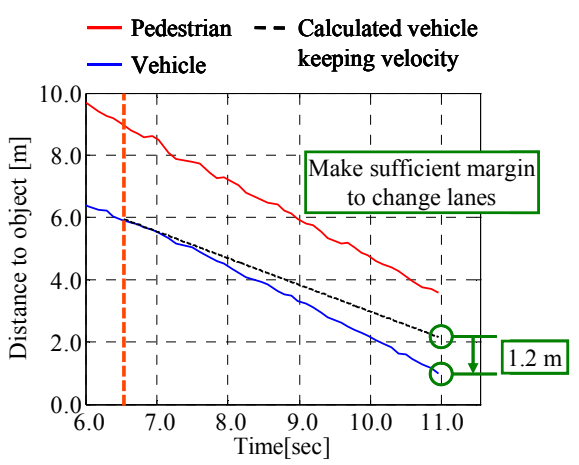

Fig. 17 Detailed transition of distance for the acceleration case of situation 2

In contrast, Fig. 18 shows the time-series experimental results for the deceleration case of situation 2. In this case, as the predicted locomotion distance of the vehicle is smaller than that of the pedestrian when the situation satisfied the conditions, the vehicle can make a sufficient margin distance by deceleration rather than acceleration. Unlike the other two cases, the pedestrian overtook the vehicle in this case. Because this vehicle temporally missed the pedestrian in the blind areas of the laser range sensors, the distances to the object could not be measured in some parts. To grasp the trend in the data, Fig. 18 shows linear interpolated data (represented by dotted lines in red and blue). Similar to the other two cases, Fig. 19 picks up the green circled part of the distance to the object shown in Fig. 18. In this case, velocity control has the effect of not only making a sufficient margin distance but also making the overtaking timing early. As this effect on the timing adjustment enables further interactions for safe locomotion, it is also important for the interaction between the vehicle and the pedestrian.

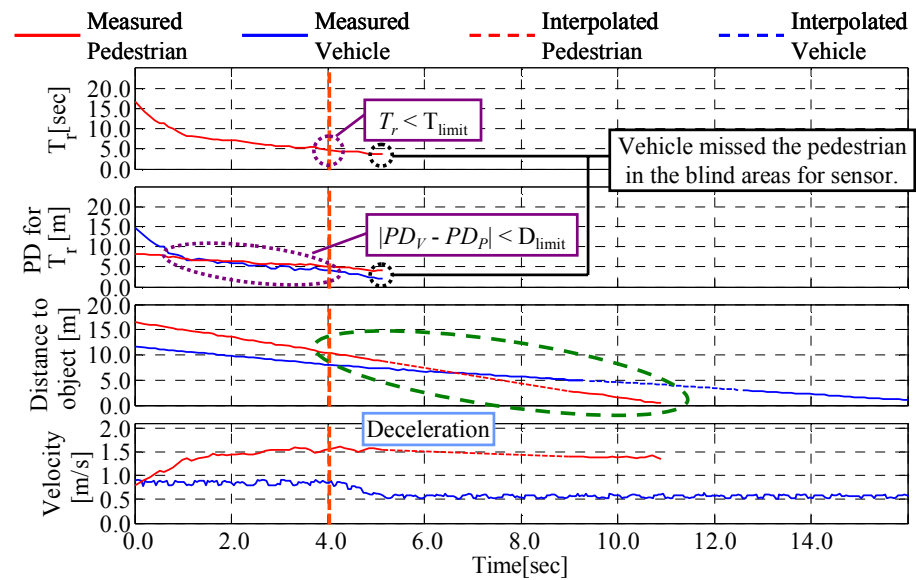

Fig. 18 Experimental results for the deceleration case of situation 2 


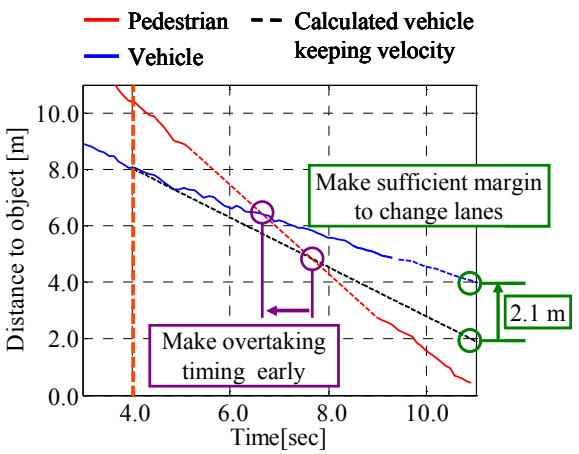

Fig. 19 Detailed transition of distance for the deceleration case of situation 2

Through the experiment, we verified the operations of the proposed method and implemented system. For all of the assumed cases, our system estimated the situations adequately and executed velocity control to enable the vehicle to make a sufficient margin to avoid a pedestrian changing lanes.

\section{Conclusions}

The objective of this study was to develop an interactive strategy of velocity control that takes into consideration the interaction prediction of surrounding the pedestrians. Based on an examination of the characteristics of sidewalks as a locomotion environment, we proposed velocity control strategy based on rough interaction prediction. The proposed method was implemented in the prototype intelligent powered wheelchair, and the operations of the proposed method and implemented system were verified. The results of the experiment revealed that the system could provide a sufficient margin for the assumed situations.

Nevertheless, much remains in this area of research. Although this study only assumed interactions between a pedestrian and a static object, interactions between several pedestrians can occur. In addition, this study ignored the interaction of another pedestrian when the vehicle accelerated or decelerated. Resolving these problems would make the system more practical and useful.

\section{Acknowledgement}

This work was supported by Grant-in-Aid for JSPS Fellows 10J08623.

\section{References}

(1) Rory, A. C., Michael, L. B., Donald, M. S., Dan, D., Songfeng, G., Alicia, M. K., Shirley, G. F., Rosemarie, C., Annmarie, K. and Diane, M. C., Engineering Better Wheelchair to Enhance Community Participation, IEEE Transactions Neural Systems and Rehabilitation Engineering, Vol. 14, No. 4(2006), pp. 438-455.

(2) Kayama, K., Yairi, E. I. and Igi, S., Outdoor Environment Recognition System on Robotic Communication Terminals Supporting Mobility of Elderly and Disabled People, Systems and Computers in Japann, Vol. 37, No. 8(2006), pp. 56-67.

(3) Matsumoto, O., Komoriya, K., Hatase, T., Nishimura, H., Toda, K. and Goto, S., Autonomous Traveling Control of the "TAO Aicle" Intelligent Wheelchair, Proceedings of the 2006 IEEE/RSJ International Conference on Intelligent Robots and Systems, (2006-10), pp. 4322-4327.

(4) Goto, T. and Yamaoka, M., Personal Mobility Robot (in Japanese), Journal of Society of Automotive Engineers of Japan, Vol. 64, No. 5(2010), pp. 75-78.

(5) Hatao, N., Hanai, R., Yamazaki, K. and Inaba, M., Real-Time Navigation for a Personal Mobility in an Environment with Pedestrians, Proceedings of The 18th IEEE International Symposium on Robot and Human Interactive Communication, (2009-9), pp. 619-626. 
(6) Morales, Y., Takeuchi, E., Carballo, A., Tokunaga, W., Kuniyoshi H., Aburadani, A., Hirosawa, A., Nagasaka, Y., Suzuki, Y. and Tsuouchi, T., 1Km Autonomous Robot Navigation on Outdoor Pedestrian Paths "Running The Tsukuba Challenge 2007", Proceedings of the 2008 IEEE/RSJ International Conference on Intelligent Robots and Systems, (2008-9), pp. 219-225.

(7) Hosoda, Y., Yamamoto, K., Ichinose, R., Egawa, S., Tamamoto, J., Tsubouchi, K. and Yuta, S., Collision-Avoidance Algorithm for Human-Symbiotic Robot, Proceedings of International Conference on Control, Automation and Systems 2010, (2010-10), pp. 557-561.

(8) Ziebart, D. B., Ratliff, N., Gallagher, G., Mertz, C., Peterson, K., Bagnell, J. A., Herbert, M., Dey, A. K. and Srinivasa, S., Planning-based Prediction for Pedestrians, Proceedings of the 2009 IEEE/RSJ International Conference on Intelligent Robots and Systems, (2009-10), pp. 3931-3936.

(9) Chen, Z. and Yung, N.H.C., Improved Multi-Level Pedestrian Behavior Prediction Based on Matching with Classified Motion Patterns, Proceedings of the 12th International IEEE Conference on Intelligent Transportation Systems, (2009-10), pp. 249-254.

(10) Ohki, T., Nagatani, K. and Yoshida, K., Collision Avoidance Method for Mobile Robot Considering Motion and Personal Spaces of Evacuees, Proceedings of the 2010 IEEE/RSJ International Conference on Intelligent Robots and Systems, (2010-10), pp. 1819-1824.

(11) Dirk, S., Wolfram, B., Dieter, F. and Armin, B. C., People Tracking with Mobile Robots Using Sample-based Joint Probabilistic Data Association Filters, The International Journal of Robotics Research, Vol. 22, No.2(2003), pp.99-116.

(12) Liu, C., Ooeda, Y. and Sumi, T., A Model for Pedestrian Movement with Obstacle Evasion Using Personal Space Concept (in Japanese), Doboku Gakkai Ronbunshuu D, Vol. 64, No. 4 (2008), pp.513-524. 